\title{
Characterization of Protein Metabolism in Undifferentiated and Differentiated Murine Muscle Tissue
}

JESSICA M. CARDIN, J. WILLIAM DEAVER, COLLEEN L. O'REILLY, STEPHEN F.
CROUSE, FASCM, AND JAMES D. FLUCKEY
Muscle Biology Lab; Department of Health and Kinesiology; Texas A\&M University; College Station, TX

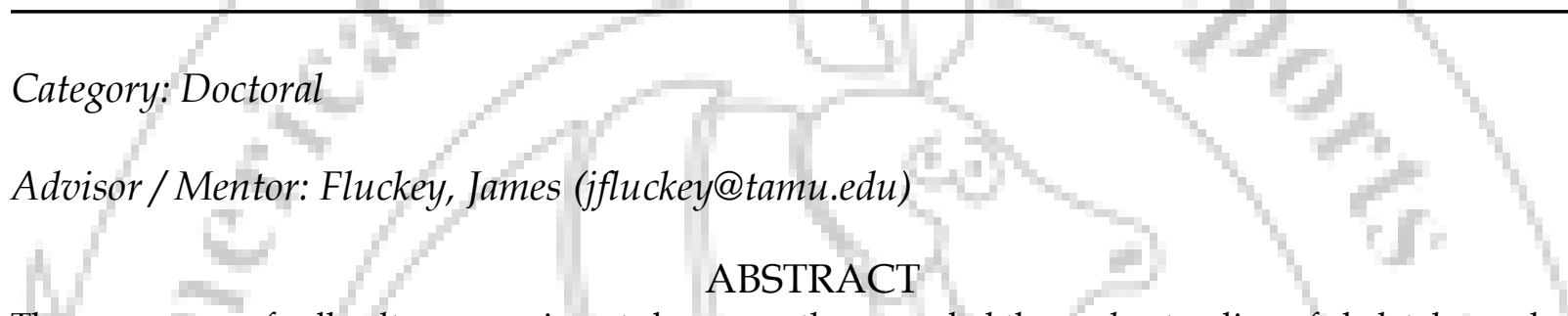

The emergence of cell culture experiments have greatly expanded the understanding of skeletal muscle physiology. However, there is a paucity of data regarding the behaviors of cells grown in culture at various stages versus in vivo. This preliminary set of studies was designed to assess alterations of anabolic responses between undifferentiated and differentiated muscle tissue in [high] and [low] glucose media along with varying dosages of insulin. Purpose: Determine if there is a disparity in fractional synthesis rates (FSR) between C2C12 myoblasts and myotubes with varying levels of insulin and in [high] $(4.5 \mathrm{~g} / \mathrm{L})$ and $[\mathrm{low}]$ glucose $(2.75 \mathrm{~g} / \mathrm{L})$ media. Methods: All cells that were going to be differentiated were started on a [high] glucose differentiation media for 48 hours. The [high] glucose differentiation media was continually applied for the [high] glucose group until harvest of the cells. The [low] glucose media group had the [high] glucose differentiation media removed and [low] glucose differentiation media was applied for 48 hours until the cells were harvested. Both [low] and [high] glucose groups received three different levels of insulin. T-25's received either $75 \mu \mathrm{L}, 150 \mu \mathrm{L}$, or $300 \mu \mathrm{L}$. T-75's $195 \mu \mathrm{L}, 390 \mu \mathrm{L}$, and 780 $\mu \mathrm{L}$. Deuterium oxide was applied 24 hours prior to harvest of the cells at a level of $4 \%$. Results: Preliminary data demonstrates that differentiated murine myotubes have slightly elevated FSR than undifferentiated myoblasts $(\mathrm{p}<0.013)$. When insulin was added to the growth media, FSR was found to be elevated in undifferentiated cells compared to controls $(\mathrm{p}<0.05)$. Within the differentiated myotubes, the [low] glucose myotubes had higher FSR than myotubes that were incubated in [high] glucose myotubes $(p<0.001)$. There was also no difference in FSR based on flask size for either the undifferentiated $(p>0.181)$ or differentiated ( $p>0.464) \mathrm{C} 2 \mathrm{C} 12$ 's. Conclusion: Future investigators must be aware of the ratio of undifferentiated cells and differentiated myotubes as this ratio could confound results as myoblasts are still present even at later stages of differentiation. Current protocols for differentiation media, regarding insulin addition, provide for optimal anabolic responses. Elevated FSR rates in the myotubes fed [low] glucose media could be explained by the cells having a higher turnover rate of cellular proteins. 